\title{
Die Meerenge von Gibraltar Küstenmorphologie zwischen Mittelmeer und Atlantik
}

Die Meerenge von Gibraltar ist ein nur $70 \mathrm{~km}$ breiter Grenzsaum zwischen zwei morphologisch völlig unterschiedlichen Küstenregionen.

Für die Mittelmeerküsten gelten:

- kleiner Tidenhub;

- eher ab- als auflandige Winde;

- meist schwache Brandung und wenig Stürme;

- kleine Geschwindigkeiten der Wasserströmungen;

- kurze Torrenten mit stark wechselnder Wasserführung.

Die Atlantikküsten werden intensiver umgestąltet als die Mittelmeerküsten und für sie gelten:

- großer Tidenhub;

- auflandige Winde fast das ganze Jahr über;

- meist starke Brandung und häufig hohe Windgeschwindigkeiten;

- stark versetzende Küstenströmungen;

- Flüsse mit großen Einzugsgebieten und hoher Sedimentzufuhr.

Für den Grenzsaum zwischen den beiden Meeren drängen sich zwei Fragen auf:

1. Wie verändern sich die küstenmorphologisch wirksamen Größen und deren Zusammenspiel zwischen Mittelmeer und Atlantik?

2. Welches sind die Auswirkungen auf die bisher entstandenen Küstenformen und die rezente Morphodynamik?

Die erste Frage konnte mittels Karten und aufgrund der vorhandenen Arbeiten über diesen Raum (siehe Literaturverzeichnis) bereits teilweise beantwortet werden. Für die Absicherung der ersten und die Beantwortung der zweiten Frage war eine Geländebegehung nötig; sie erfolgte im März 1979.

Das Arbeitsgebiet (Abb. 1) umfaßt die iberische Seite der Meerenge von Europe Point (Gibraltar) bis Cabo Trafalgar (zwischen Barbate und Conil) und die afrikanische zwischen Punta Almina (Ceuta) und Cap Spartel (westlich von Tanger). Für einzelne Aspekte wurden auf der europäischen Seite Beobachtungen zwischen Almería (Südostspanien) und Carrapeteira (Südwestportugal) mit einbezogen.

Ludwig Ellenberg, Institut für Geographie. Technische Universität Berlin, D-1 Berlin 12.
1. Wie verändern sich die küstenmorphologisch wirksamen Größen und ihr Zusammenspiel zwischen Mittelmeer und Atlantik?

\subsection{Tidenhub und Gezeitenströmung}

Der Tidenhub im Mittelmeer ist klein. Der mittlere Tidenhub der im allgemeinen halbtägigen Gezeit steigt nur in drei Gebieten über $50 \mathrm{~cm}$ : in der nördlichen Adria, im Golf von Gabès und im Bereich der Straße von Gibraltar.

Am Ostausgang der Meerenge beträgt der Springtidenhub etwa $1 \mathrm{~m}$, an den atlantischen Küsten bereits über $3 \mathrm{~m}$.

$\begin{array}{llr} & \text { Springtidenhub } & \begin{array}{c}\text { Spm } \\ \text { cm }\end{array} \\ \text { Osten } & \text { Restinga } & 80 \\ & \text { Ceuta } & 110 \\ & \text { Gibraltar } & 100 \\ & \text { Algeciras } & 120 \\ \text { Mitte } & \text { Tarifa } & 180 \\ & \text { Tanger } & 250 \\ \text { Westen } & \text { Cap Spartel } & 300 \\ & \text { El Araich } & 330 \\ & \text { Conil } & 340 \\ & \text { Cadiz } & 390\end{array}$

In ihrer Richtung wechselnde Gezeitenströmungen fließen entlang aller Küsten. Im Osten sind sie ohne große Auswirkung auf die Morphodynamik, in der Meerenge selbst und im Westen, wo sie $150 \mathrm{~cm} / \mathrm{sec}$ oft übersteigen, sind sie für Abtragung, Verfrachtung, Anlagerung und damit für einen Ausgleich im Küstenverlauf von Bedeutung.

\subsection{Wellen}

Nur die Winde aus westlichen Richtungen erreichen im Bereich der Meerenge hohe Geschwindigkeiten. So sind die Küsten des südlichen Spaniens und nördlichen Marokkos in Luv- und Leeküsten unterschieden. Die Luvküsten reichen von Westen her bis Cap Spartel und Tarifa, abgeschwächt bis Punta Almina und Europe Point. An ihnen bestimmt die Brandung die Morphodynamik. Der Einfluß auf das Hinterland, beispielsweise durch Dünen oder Salzspray, ist 


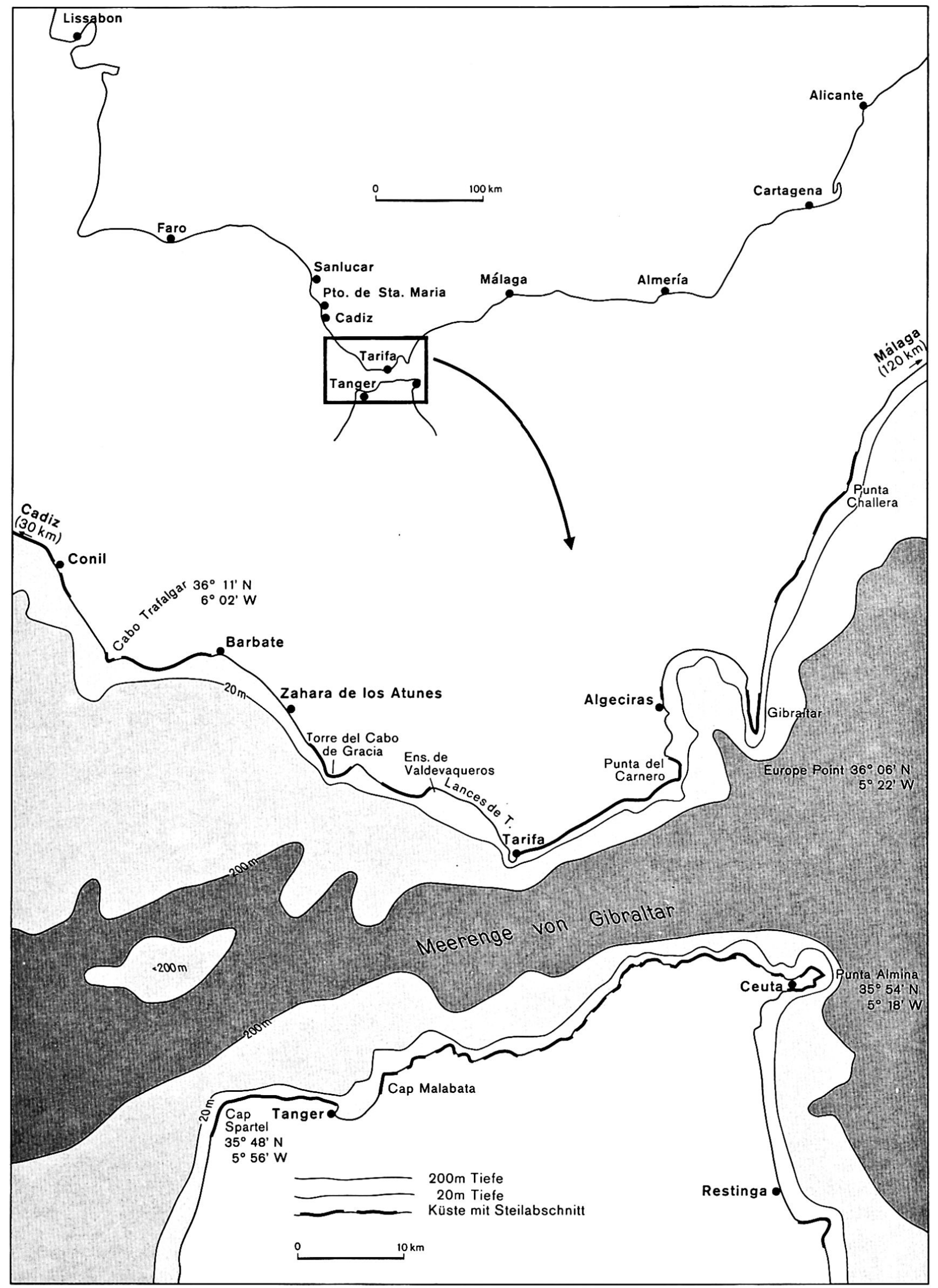

Abb. 1: Untersuchungsgebiet 


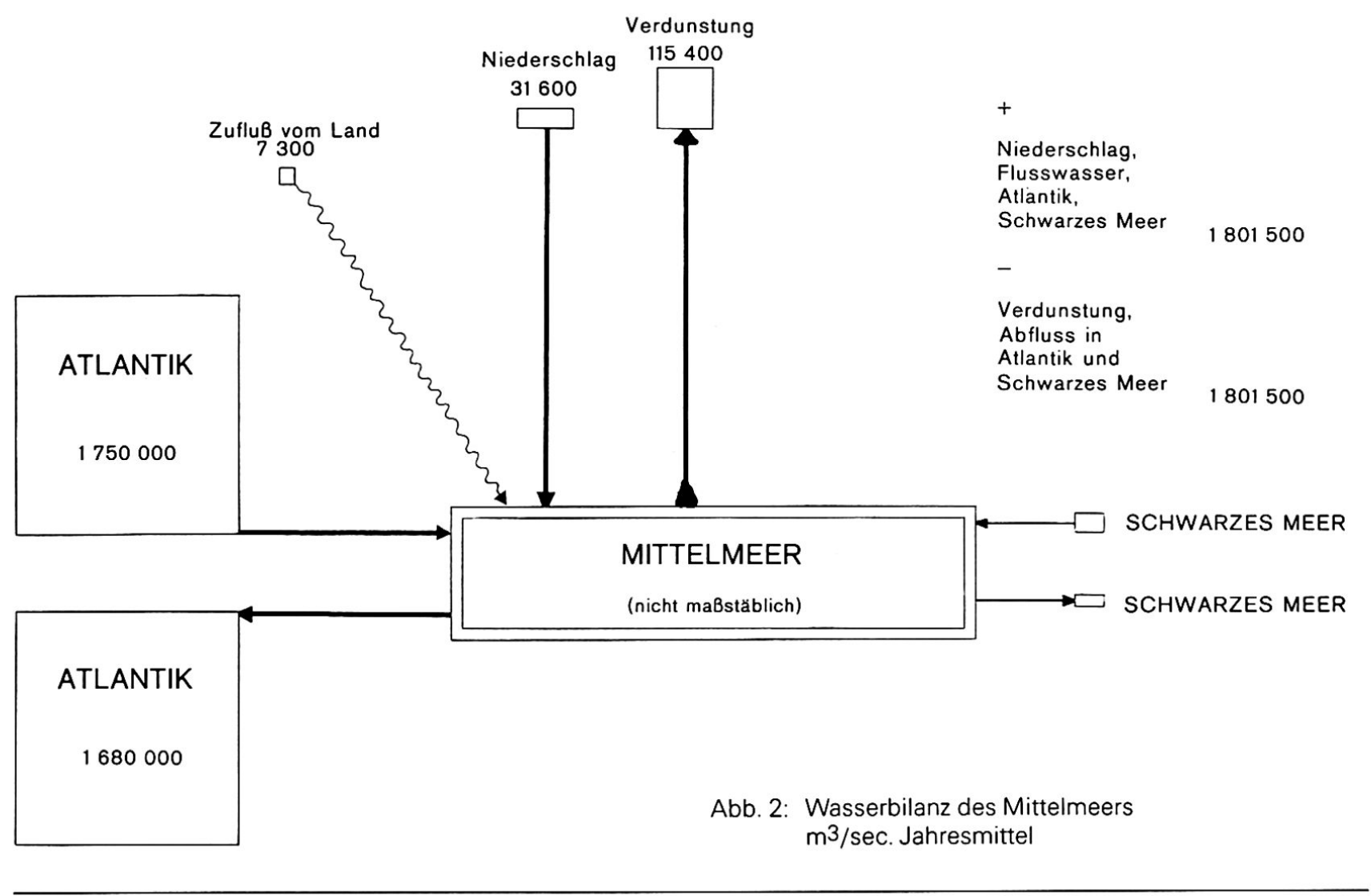

im Gegensatz zu den Mittelmeerküsten nicht nur ein schmaler Saum.

Die Veränderung im Brandungsregime vollzieht sich abrupt; der Atlantik kann auf diese Weise vom Mittelmeer abgegrenzt werden. In Europa liegt die Grenze in der Mitte der Meerenge (Tarifa), in Afrika am Westausgang (Cap Spartel).

Winde über $11,3 \mathrm{~m} / \mathrm{sec}$ (22 Knoten) kommen selten zwischen Juni und August vor und Stürme mit Geschwindigkeiten über $17,5 \mathrm{~m} / \mathrm{sec}$ (34 Knoten) ausschließlich zwischen Dezember und April (im März im Mittel an drei Tagen).

\subsection{Küstenströmung}

Windinduzierte Strömungen sind überall außer in engen Buchten zu spüren. Sie sind nicht richtungskonstant, doch sind die den Küsten vom Atlantik zum Mittelmeer folgenden die bestimmenden; ihre Geschwindigkeit kann $1 \mathrm{~m} / \mathrm{sec}$ übersteigen.

Trockenheit, relativ geringer Zufluß vom Land her und hohe Verdunstung würden den Spiegel des Mittelmeeres in jedem Sommer weiter absenken, wenn nicht Atlantik- und Schwarzmeerwasser das Defizit decken könnten (Abb. 2).

Der außerordentlich große Zufluß vom Atlantik (240 mal soviel, als dem Mittelmeer durch Flüsse zugeführt wird!), der im Gegensatz zum salzreicheren und deshalb schwereren Gegenstrom in den oberen Wasserschichten erfolgt, bedingt unabhängig von Wind und Gezeiten eine nach Osten versetzende Küstenströmung, die $250 \mathrm{~cm} / \mathrm{sec}$ erreichen kann.

\subsection{Zusammensetzung des Meerwassers und Temperaturen}

Der Salzgehalt des Oberflächenwassers ist normal und schwankt nur wenig. Er beträgt im Osten $3,65 \%$, sinkt gegen Westen ab und nimmt mit der Tiefe zu ( $3,8 \%$ bei $600 \mathrm{~m}$ Tiefe).

Den intermediären subtropischen Jahresgang der Temperaturen veranschaulicht Abb. 3. Die zu erkennende Zunahme der Temperaturschwankungen von Westen nach Osten ist zu klein, um sich küstenmorphologisch auszuwirken.

\subsection{Einflüsse von Organismen an der Küste}

Hierin besteht kein krasser Gegensatz zwischen Atlantik und Mittelmeer. Für das Wachstum von Korallen sind die Wassertemperaturen zu niedrig. Beach rock tritt im Arbeitsgebiet nicht auf. Bioerosion ist im Bereich des Wasserspiegels besonders an Kalkküsten aktiv. Lösungskehlen ohne starke Ergänzung durch Brandungsprozesse gibt es nicht; sie sind eher für Ostspanien typisch. 


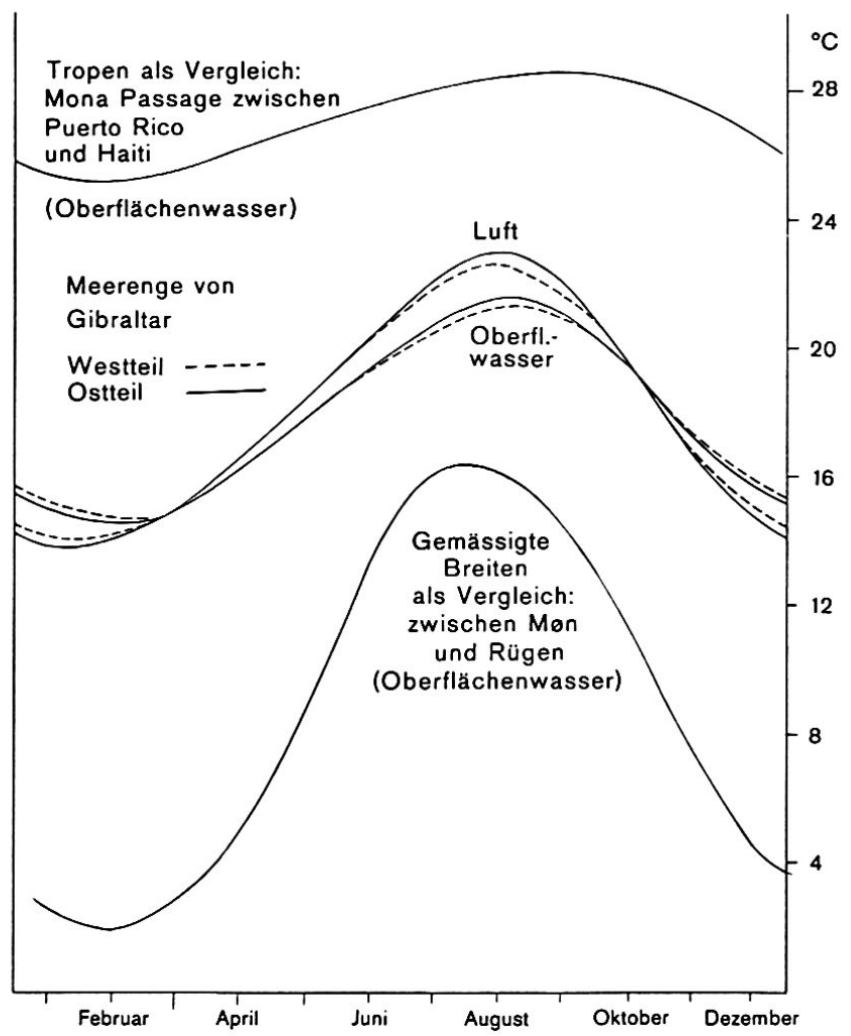

\subsection{Terrestrische hydrologische Bedingungen und terrestrische morphologische Formung}

Im Bereich der Meerenge von Gibraltar sind die Einzugsgebiete im Osten (z. B. Rio Guardiaro) nur wenig kleiner als im Westen (z. B. Rio Barbate). Von Almería ab gegen Westen werden den Küsten ständig Sedimente vom Land her zugeführt. Am stärksten ist dies im weiteren Bereich der Meerenge in den folgenden drei Regionen der Fall (Abb. 4): nordöstlich von Gibraltar, in der Bahía de Algeciras und im Mündungsgebiet des Rio Barbate. Lediglich die kurzen Bäche zwischen Algeciras und dem Rio Barbate sind nur unmittelbar nach Regenfällen aktive Sedimentzulieferer.

Nördlich von Cadiz, bei Sanlúcar de Barrameda, mündet der Guadalquivir $\left(57000 \mathrm{~km}^{2}\right.$ großes Einzugsgebiet, $560 \mathrm{~km}$ Länge, $164 \mathrm{~m}^{3} / \mathrm{sec}$ übers Jahr gemittelte Wasserführung). Er bestimmt die küstenmorphologischen Verhältnisse auf große Distanz. Mit ihm setzt auch das atlantische Regime der Flüsse Iberiens ein.

$\triangleleft$ Abb. 3: Temperaturverhältnisse

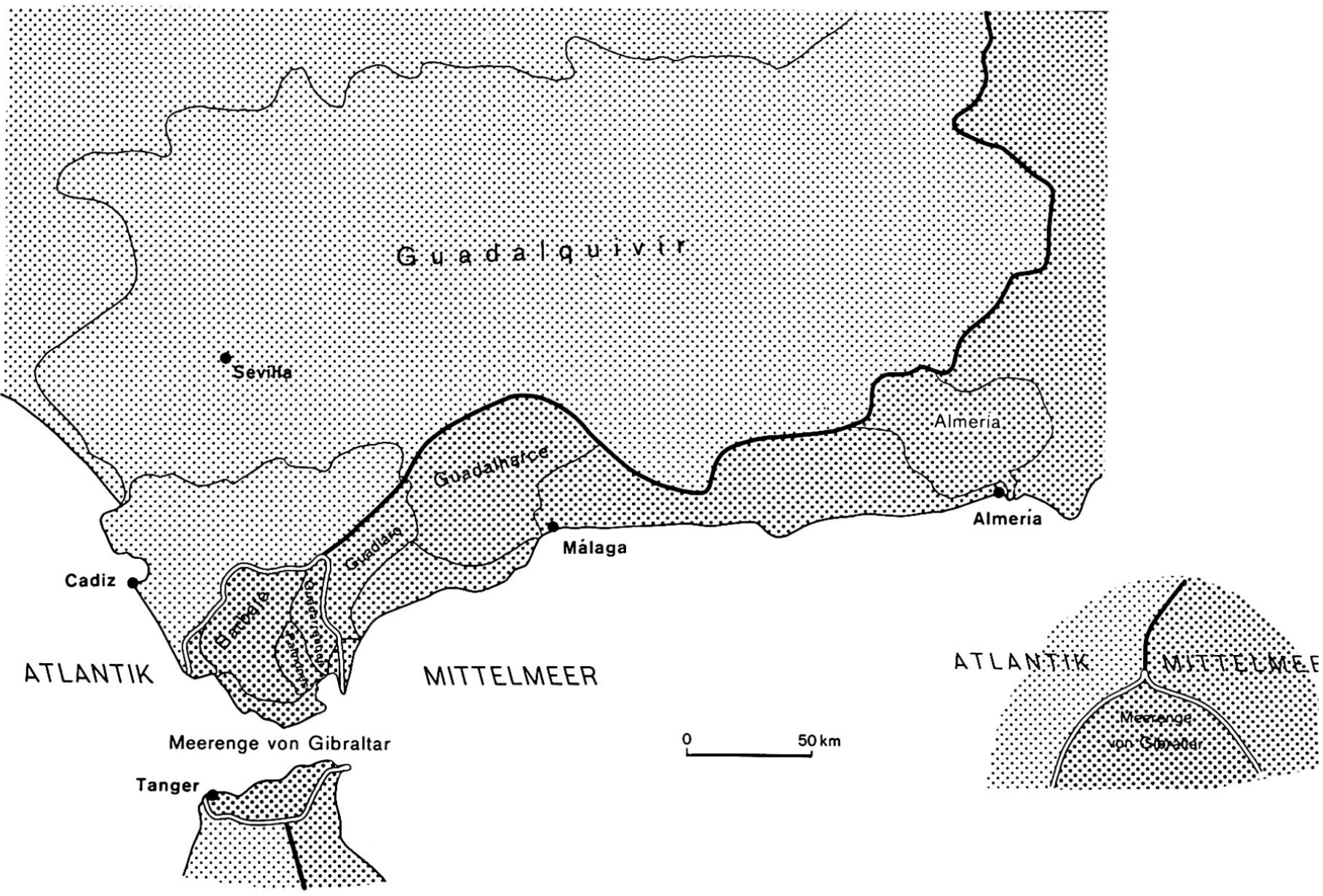

Abb. 4: Einzugsgebiete der Flüsse 
Da die Wasserscheide zwischen Mittelmeer und Atlantik die iberische Halbinsel nicht in der Mitte durchzieht, sondern stark nach Osten versetzt ist, sind alle großen Flüsse außer dem Ebro nach Westen gerichtet und münden in Südwestspanien und Portugal. Die Asymmetrie hat weitreichende Konsequenzen: ins Mittelmeer werden durch zumeist kurze, steil angelegte Torrenten während der Wintermonate oder nach sommerlichen Gewittern Geröll und Sand grober Fraktionen zugeführt; während der übrigen Zeit des Jahres liegen die Ramblas trocken und vertriftetes Strandmaterial versperrt die Mündung. In den Atlantik fließen die mittelgroßen und großen Flüsse ganzjährig; ein Mündungsverschluß ist höchstens zeitweise angedeutet. Große Mengen feinkörnigen Materials werden den Küsten zugeliefert, in Form breiter Strände, Buchtfüllungen und Nehrungen angelagert, versetzt und vom Wind wieder landeinwärts verweht. Dieser Unterschied ist wie erwähnt im südlichsten Iberien stark abgeschwächt. Auf der marokkanischen Seite ist das Einzugsgebiet der Meerenge schmal (max. Breite $25 \mathrm{~km}$ ). Die kurzen Bäche führen episodisch bis periodisch grobes Material dem Meer zu. Einzig in der Bucht von Tanger gibt es größere Feinmaterialakkumulationen.

Die terrestrische Formung an Steilhängen im Küstenbereich ist in diesem außer Galizien regenreichsten Gebiet Tiefland-Iberiens relativ aktiv. Flächenhaft wirkende und prägnant linienhafte Abtragung mit Zerschluchtung - besonders stark in den Flyschgesteinen zwischen Punta del Carnero und Torre del Cabo de Gracia sind neben Windprozessen auf der spanischen Seite bedeutsam. Auf der marokkanischen verändern sich die Kalkhänge relativ langsam; am aktivsten sind hier die steilen Ufer zwischen Tanger und Cap Spartel, wo sich in Kreide-Flysch viele Rutschungen und Sackungen lösen.

Die kühleren Monate mit häufigen und heftigen Regenfällen (Gibraltar Oktober bis März $630 \mathrm{~mm}$ ) sind dabei entscheidender als der Sommer (Gibraltar April bis September $130 \mathrm{~mm}$ ). Spülprozesse und Rutschungen kommen in den Sommermonaten fast ganz zur Ruhe und Deflationsprozesse sind wegen geringerer Geschwindigkeiten der Winde nur abgeschwächt wirksam.

\section{$1.7 \mathrm{Eu}$ - und isostatische Niveauveränderungen}

Spuren höherer quartärer Meeresstände sind an den Küsten und entlang der Flüsse fast durchgehend festzustellen. Am Felsen von Gibraltar sind Terrassenreste in $8,16,23,38,83$ und $123 \mathrm{~m}$ Höhe zu finden. Die Maximalhöhen der heute in Formung begriffenen Kliffs liegen auf der spanischen Seite meist unter $20 \mathrm{~m}$ und sind durch Abrasionsplattformen in verschieden hohen Niveaus vorgegeben. Die isostatischen Bewegungen scheinen seit dem Mindel zur Ruhe gekommen zu sein (G. GIERMANN, 1962).

\subsection{Anthropogene Einflüsse}

Die direkten Eingriffe des Menschen durch Bauten an den Küsten (Molen, Häfen, Uferschutz, Kanalisierungen von Flußmündungen) sind an der mittelmeerischen Seite Iberiens wesentlich umfangreicher als an der atlantischen. Im Bereich der Meerenge kann die Bahía de Algeciras mit der Westseite von Gibraltar als fast völlig verbaut gelten, während westlich davon nur wenige punktförmige Eingriffe erfolgten. Auf der marokkanischen Seite sind Küstenbauten auf Ceuta und den Westteil der Bucht von Tanger beschränkt.

Groß ist hingegen der indirekte anthropogene Einfluß, der sich über die Veränderung von Pflanzendecke, Landnutzung und Böden auf die hydrologischen Bedingungen auswirkt. Von Natur aus würden die Abflußspitzen der Flüsse weniger extrem und dafür über einen längeren Zeitraum auftreten und damit kurzzeitig starke Sedimentschüttungen ins Meer seltener und schwächer sein.

Abb. 5 zeigt schematisch, daß sich die rezente Morphodynamik der Küsten im atlantischen Teil der Meerenge im allgemeinen rascher und tiefgreifender vollzieht und mehr vom Meer her bestimmt wird als im mittelmeerischen.

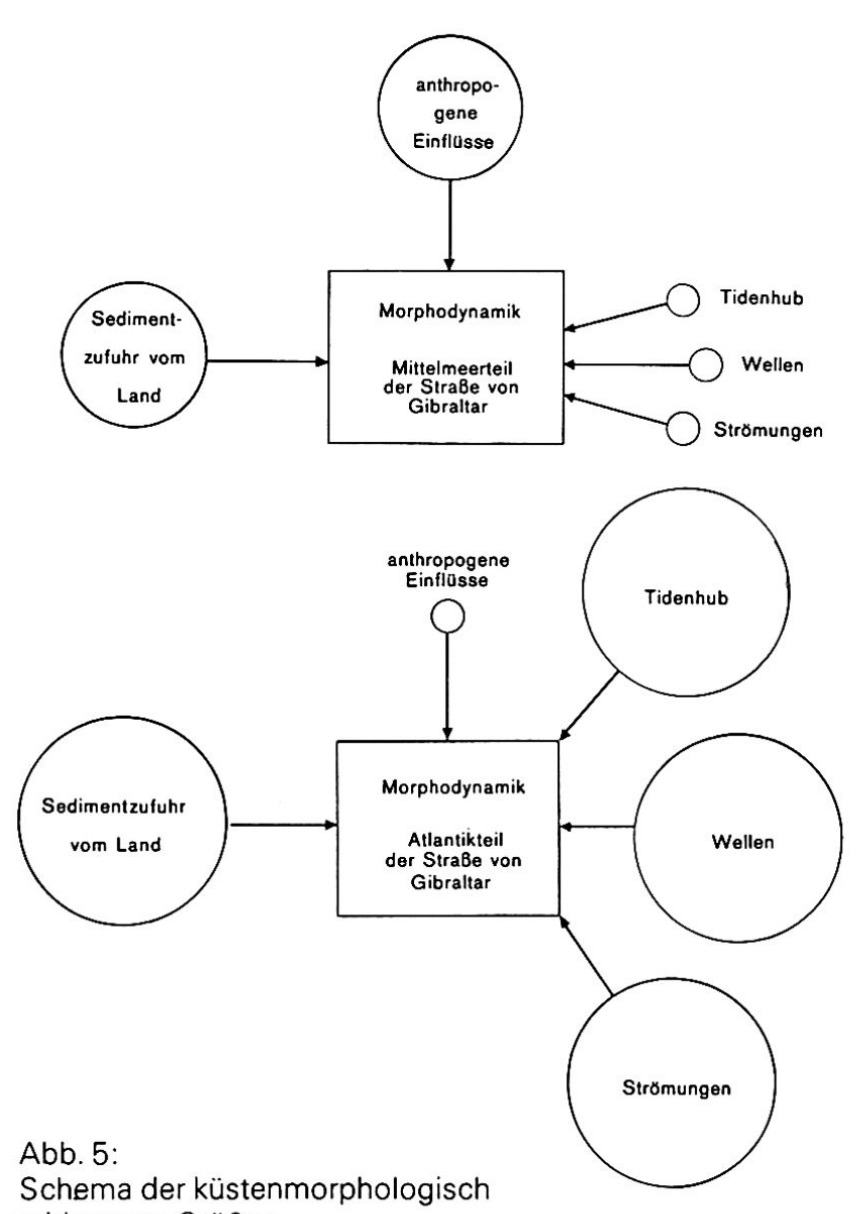
wirksamen Größen 


\section{Welches sind die Auswirkungen auf die rezente Morphodynamik und die bisher entstandenen Küstenformen?}

Die aufgrund der bisherigen Ausführungen zu erwartenden Modifikationen der Küsten zwischen Mittelmeer und Atlantik werden an den beiden Seiten der Meerenge veranschaulicht.

\subsection{Europäische Seite der Straße von Gibraltar}

Da der Wechsel von mittelmeerisch östlich zu atlantisch westlich exponierten Küsten nicht abrupt erfolgt, sind die atlantischen Elemente bei einem Gang von Osten nach Westen zunächst kaum zu erkennen, werden ab Punta del Carnero deutlich und dominieren ab Tarifa.

\subsection{Mittelmeerwärts von Gibraltar}

Zwischen Málaga und Gibraltar - deutlich z. B. bei Punta Chullera - sind Abrasionsplattformen entstanden, an denen gekappte härtere Schichten als vorspringende Zähne 100 und $200 \mathrm{~m}$ weit ins Meer hinausragen. Diese Form der Abrasionsplattformen ist an der spanischen Mittelmeerküste sonst nicht zu finden.

Deltas, die in Ost- und Südostspanien auch bei kleinen Flüssen aufgebaut werden, fehlen ab Málaga fast vollkommen; zu stark scheint der Versatz durch die Küstenströmung zu werden.

Bereits östlich von Málaga setzen gleichmäßig abgedachte Sandstrände ein, auf denen sich schmale Spülsäume, verursacht durch das Auslaufen der höchsten Wellen bei wechselnden Wasserständen, erkennen lassen. Gegen Süden werden die Höhenunterschiede zwischen Ebbeniveau und trockenem Strand oder Strandwall rasch größer. An den schließlich unübersehbar tidebeeinflußten Küsten müssen die Fischerboote hoch auf den trockenen Strand gezogen werden und können nicht mehr so einfach wie in Ostspanien am Ufer vertäut werden.

Während Dünen die Mittelmeerküsten Spaniens selten begleiten (an weniger als 10\% der aufgesuchten Lokalitäten zwischen der französischen Grenze und Málaga), werden sie gegen Süden immer häufiger (an 16\% der aufgesuchten Lokalitäten zwischen Málaga und Gibraltar) und dabei höher, aktiver und weiter ins Land hineinreichend. Wie eine Vorschau auf atlantische Verhältnisse muten die Küsten um Marbella an, wo ausgehend von einem $4 \mathrm{~m}$ hohen Dünenwall am Ufer landeinwärts ein Sandschleier über einen sanft ansteigenden Hang 300 m landeinwärts verweht wurde.

An der Ostseite des Kalkklotzes von Gibraltar, der im Gegensatz zur Westküste noch natürliche Küsten zeigt, sind in die steilen Felsflanken mehrere Meter tiefe und über $2 \mathrm{~m}$ hohe Hohlkehlen eingefressen, die eher lösungs- als brandungsbedingt erscheinen. Verglichen mit entsprechenden Formen in Nordostspanien (z. B. Cala de Montogo, Garraf) oder zwischen Valencia und Alicante (z. B. Denia, Javea, Calpe) verdoppelt sich die Höhe dieser Formen, was auf die vergrößerte Wasserschwankung zurückgeführt wird.

\subsection{Bahía de Algeciras}

Die Bucht zwischen Gibraltar und Punta del Carnero reicht $10 \mathrm{~km}$ ins Land hinein; sie ist windabgeschirmt. Die Küsten wirken - wo sie nicht verbaut sind - wie stille Ufer eines Sees. Dieser mittelmeerische Eindruck wird allerdings durch drei Besonderheiten abgeschwächt.

Erstens dient die Bucht als Sammelbecken für zwei perennierende Flüsse (Guadarranque, Palmones) und mehrere Bäche mit feinkörnigen Sedimentschüttungen. Zweitens fallen bei Ebbe am Nordende der Bucht und südlich von Algeciras große Wattflächen trocken. Drittens umläuft eine starke Gezeitenströmung die Bucht im Gegenuhrzeigersinn, die für die Verlagerung von Sedimenten sorgt, die Uferlinie in ihrem Verlauf ausgleicht und nördlich von Algeciras zur Inaktivierung von Kliffs durch Akkumulation von Schwemmlandsäumen geführt hat.

\subsection{Punta del Carnero bis Tarifa}

Die atlantischen Elemente mehren sich in diesem $20 \mathrm{~km}$ langen Küstenabschnitt. Es sind dünenlose Steilküsten mit etwa $20 \mathrm{~m}$ hohen Kliffs, die in küstenparallel streichenden und steil gegen die Meerenge einfallenden Mergeln, Tonen und unreinen Kalken tertiärer Flysch-Serien entstanden sind.

Eine 30-60 m breite Fels-Abrasionsterrasse (Foto 1) gehört durchgehend zur Küste. Bei Ebbe fällt sie trocken; bei Flut wird sie bis auf wenige Klippen wasserbedeckt und wellenbedrängt. Die Schichten werden gemäß ihrer Resistenz unterschiedlich rasch abradiert, so daß ein Schichtstufenrelief en miniature entstanden ist. Auf der Abrasionsfläche wird ein Teil der Wellenenergie umgesetzt; das Anbranden an den Kliff-Fuß bleibt bei Hochwasser aber stark genug, um die Wand zu unterspülen. Hohlkehlen, Auskolkungen und nachgebrochene Sturzmassen sind Indizien für die sich aktiv ins Hügelland hineinfressende Küste.

Bei Stürmen werden in Nischen Geröllpackungen bis $160 \mathrm{~cm}$ über dem Hochwasserniveau geschüttet. Herausgewaschene Grundmauern (Foto 2) und teilweise welleneingerissene Hauswände von Gebäuden, die nicht älter als 40 Jahre sein können, sind in der Meerenge erstmals $2 \mathrm{~km}$ östlich von Tarifa zu finden. $\mathrm{Da}$ dies nicht nur als Lee-Erosion hinter der Mole von Tarifa zu werten ist, verdeutlichen die auch weiter östlich auftretenden jungen Landverluste, dokumentiert durch Reste von Weidezäunen, die 


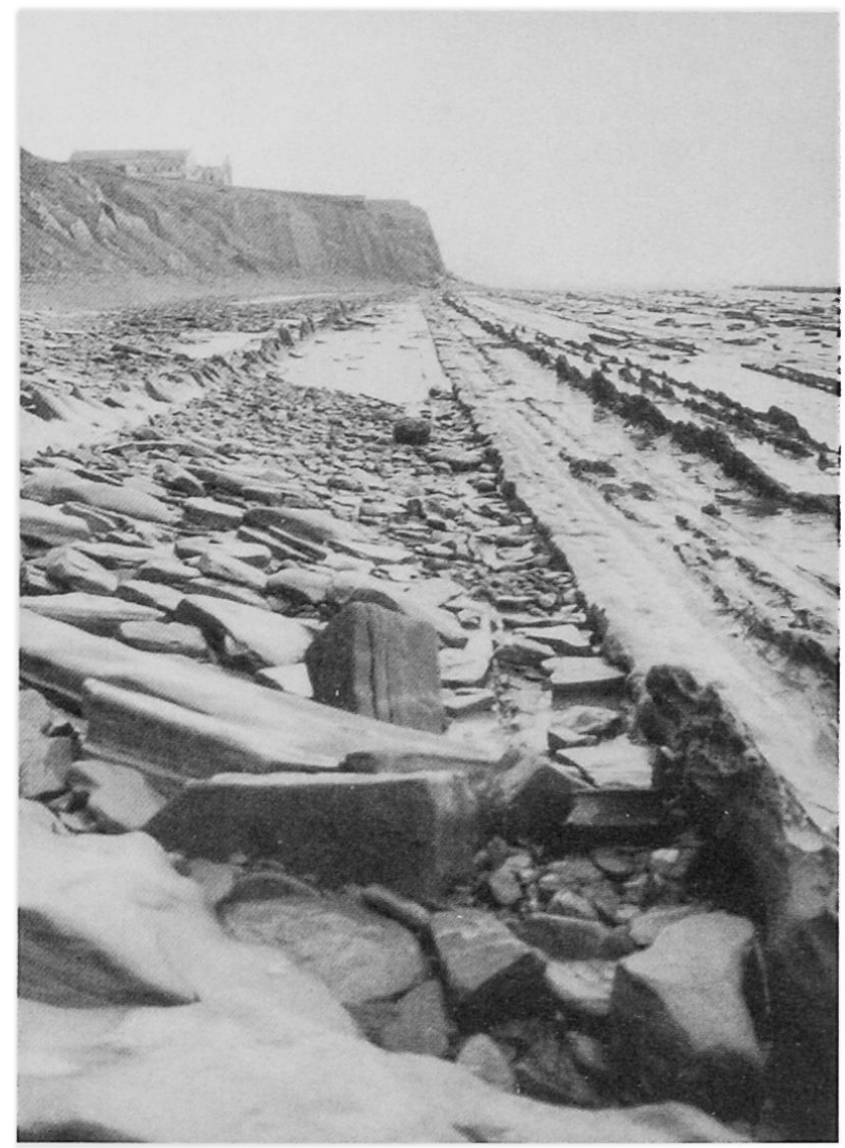

Foto 1: Abrasionsterrasse in steil einfallendem Flysch östlich von Tarifa.

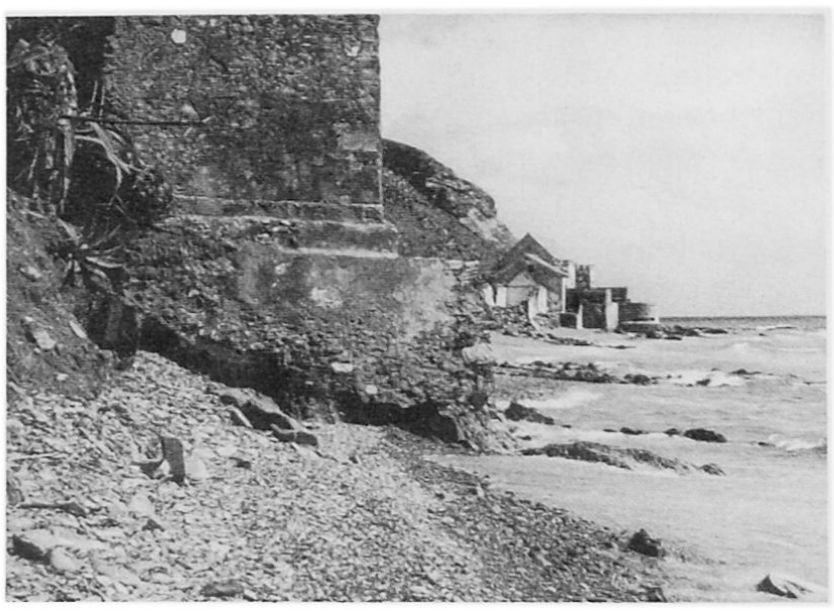

Foto 2: Wellenbedrängte Gebäude am östlichen Ortsausgang von Tarifa.

über Zehner von Metern in der Luft hängen oder noch an einzelnen Stellen erhalten geblieben sind. Die Kliff-Hänge sind wegen der ab Punta del Carnero einsetzenden Gischt und des ins Land hineinwehenden Salzsprays meist wenig bewachsen. Außerdem wird alles verwitterte Feinmaterial abgespült oder dem Kliff-Fuß in Form von Rutschungen zugeleitet.

Foto 3: Bachmündung östlich von Tarifa. $\nabla$

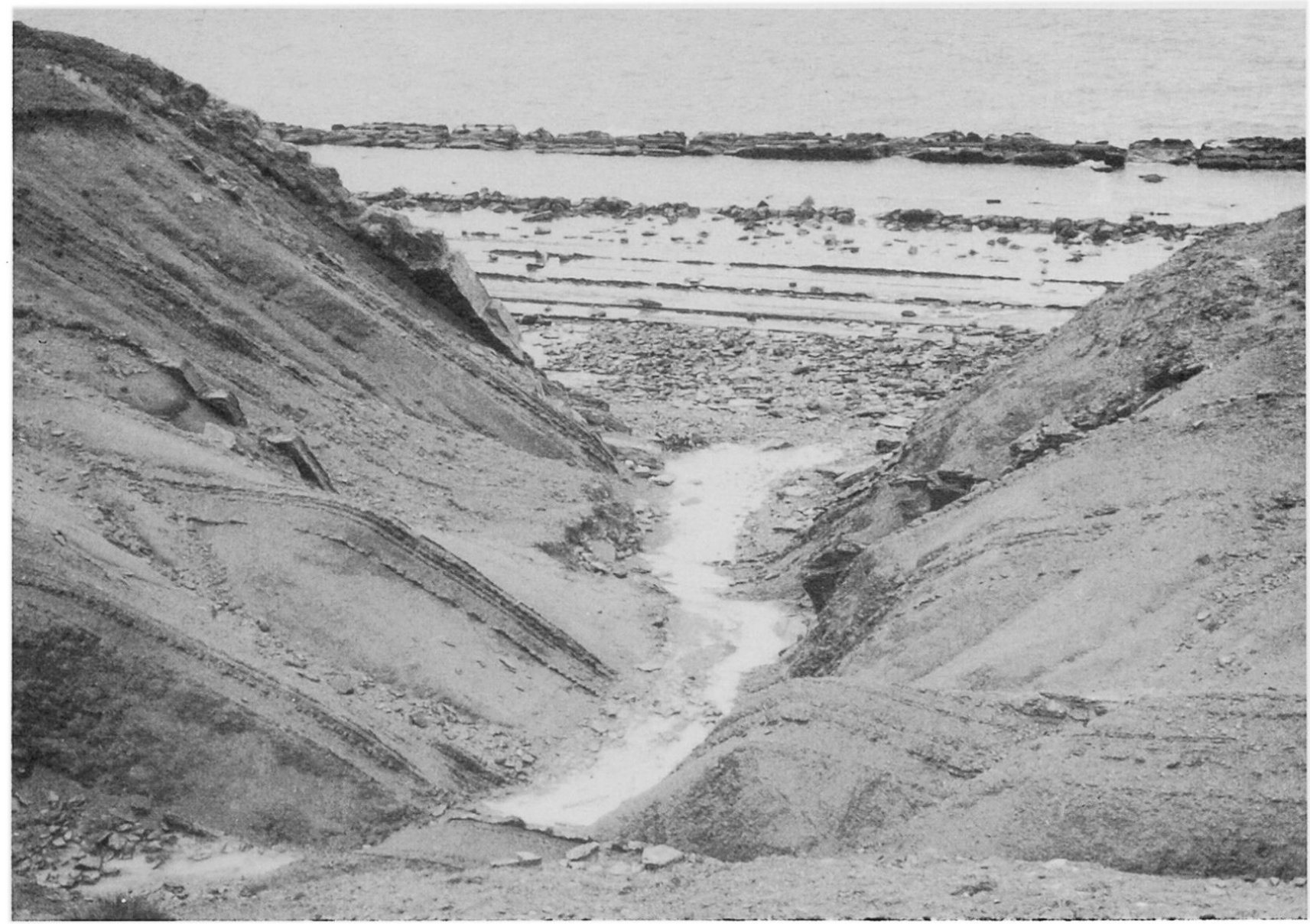


Der Landverlust wird nirgends durch Akkumulation aufgefangen. Zwar münden mehrere quer zum Schichtstreifen eingetiefte Bäche mit schluchtartigen Auslässen (Foto 3), die zugeführten Sedimente sind aber nur instabile Schleier von Feinmaterial auf der Brandungsfläche, die während des nächstfolgenden Sturms aufgegriffen und versetzt werden.

Folgt man den Küsten Iberiens von der Costa Brava im Nordosten zur Costa de la Luz im Südwesten Spaniens, so kann der Abschnitt zwischen Punta del Carnero und Tarifa als der erste dauernd stark erosiv gestaltete gelten.

\subsection{Tarifa bis Zahara de los Atunes}

$\mathrm{Ab}$ diesem $30 \mathrm{~km}$ langen Abschnitt dominieren die atlantischen Elemente.

Am Ortsausgang von Tarifa beginnt als Füllung einer sanften Einbuchtung im Hügelland eine $6 \mathrm{~km}$ lange Flachküste (Lances de Tarifa). Sie ist die erste typische atlantische Flachküste auf dem Weg von Osten nach Westen und unterscheidet sich von den mittelmeerischen durch eine Reihe von Merkmalen. Erstens ist der nach Osten gerichtete Versatz im Strandbereich viel deutlicher als weiter östlich. Zweitens erfolgt der etwa $5^{\circ}$ steile Strandanstieg auf mindestens $3 \mathrm{~m}$ über das Niedrigwasserniveau und es ist ein durchgehender Strandwall ausgebildet. Drittens ist der obere, meist trockene und gänzlich unbewachsene Strand breit; die Werte variieren zwischen 70 und $150 \mathrm{~m}$. Viertens fegt bei den stetigen Winden Sand über den Strand und kleine, instabile Anhäufungen sind entstanden. Fünftens ist die Mündung des Rio de la Jara mehrere $100 \mathrm{~m}$ verschleppt, verläuft am Ufer strandwallparallel und ist nur andeutungsweise verschlossen.

In der Ensenada de Valdevaqueros sind mehrere Meter hohe, sehr mobile Dünen nicht nur am Strand, sondern auch bis $30 \mathrm{~m}$ hügelan als Sanddecken aufgeweht. Solche Erscheinungen gibt es auf der Mittelmeerseite nur als Ausnahme, gegen Westen aber werden sie zur Regel, wenn genügend Sand angeliefert wird und die Küste nicht windabgewandt verläuft. $12 \mathrm{~km}$ weiter westlich, bei Torre del Cabo de Gracia, sind solche Anwehungen bis $50 \mathrm{~m}$ über dem Wasserniveau zu finden.

An den Steilküsten, die teilweise ähnliches Gepräge wie zwischen Punta del Carnero und Tarifa zeigen, sind Sand-und Geröllpackungen bis $250 \mathrm{~cm}$ über dem Hochwasserniveau nicht selten.

In der Luft hängende Weidezäune kommen gegen Westen immer häufiger vor. Neu sind vollständig von den Wellen freigelegte Bunker, die im 2. Weltkrieg in den Steilhängen an den Küsten gebaut wurden. Von den Bunkern wurden große Teile abgerissen und verschleppt.

Bei starkem Wellenangriff und in den ersten Stunden nach dem Hochwasserstand im Strandkörper ablaufendes Wasser führt zu zentimeter- bis dezimetertiefem Absacken des Sandes, der ins Meer zurückfließt. Die steilwandigen Formen erinnern an modellartig verkleinerte Anrisse von Wadis in feinkörnigem Substrat. Weiter östlich sind diese Kleinformen im Strand fast nirgends zu beobachten, gegen Westen aber treten sie regelhaft auf.

Vor Zahara de los Atunes fielen mir das erste Mal kippende und nicht nur in sich zusammenstürzende Brecher auf; am Strand waren in regelmäßigen Abständen von 50-70 m beach cusps ausgebildet.

\subsection{Mündung des Rio Barbate}

Der Rio Barbate unterscheidet sich von allen weiter östlich entwässernden Flüssen durch folgende Merkmale:

1. Der Fluß führt ganzjährig Wasser und seine Mündung wird nie verschlossen; er ist im Gegensatz zu den Flüssen an der Mittelmeerseite (Ausnahme Ebro) nicht zu durchwaten. Der Fischerhafen liegt nicht an der Küste selbst, wie es am Mittelmeer der Fall wäre, sondern $2 \mathrm{~km}$ flußauf.

2. Der Fluß durchzieht ein von ihm aufgeschüttetes Schwemmland, in dem Sümpfe erhalten geblieben sind, die an die Marismas des Guadalquivirs erinnern.

3. Die Überbrückung des Flusses ist aufwendiger als weiter mittelmeerwärts und erfolgt mit einigem $\mathrm{Ab}$ stand im Hinterland und nicht wie weiter östlich üblich an der Mündung selbst.

4. Der Tidenhub (3 m) ist nicht nur an den Küsten deutlich zu erkennen, sondern wirkt sich auch mehrere Kilometer weit ins Mündungsgebiet hinein aus durch ein Hoch- und Niedrigwasserniveau des Hauptbettes, der Seitenarme und der in den Sümpfen angelegten Entwässerungsrinnen.

5. Die Mündung ist im breiten Ufersaum nicht nur einige $100 \mathrm{~m}$, sondern $1,5 \mathrm{~km}$ nach Osten verschleppt.

\subsection{Westlich von Barbate}

Die genannten atlantischen Küstenmerkmale werden westwärts von Barbate $\left(5^{\circ} 55^{\prime} \mathrm{W}\right)$ immer häufiger. Darüber hinaus kommen noch sieben weitere dazu. Mit der Lokalität ihres ersten Auftretens seien sie genannt:

Westlich von Barbate (Foto 4) und verstärkt bei Conil de la Frontera $\left(6^{\circ} 10^{\prime} \mathrm{W}\right)$ treten lange hohe Kliffreihen auf. Die in Sandstein und Mergel hineingefressenen Kliffs sind bis über $40 \mathrm{~m}$ hoch, steilwandig und zum Teil wegen tief in den Kliff-Fuß geschlagener Brandungshohlkehlen überhängend. Die Rückverlegung ist äußerst aktiv. Sie erfolgt durch Nachbrechen, Rutschen und engmaschige Runsenspülung. Bunker sind nicht nur teilweise sondern ganz freigespült. Wracks von Fischerbooten findet man häufiger als weiter östlich. 


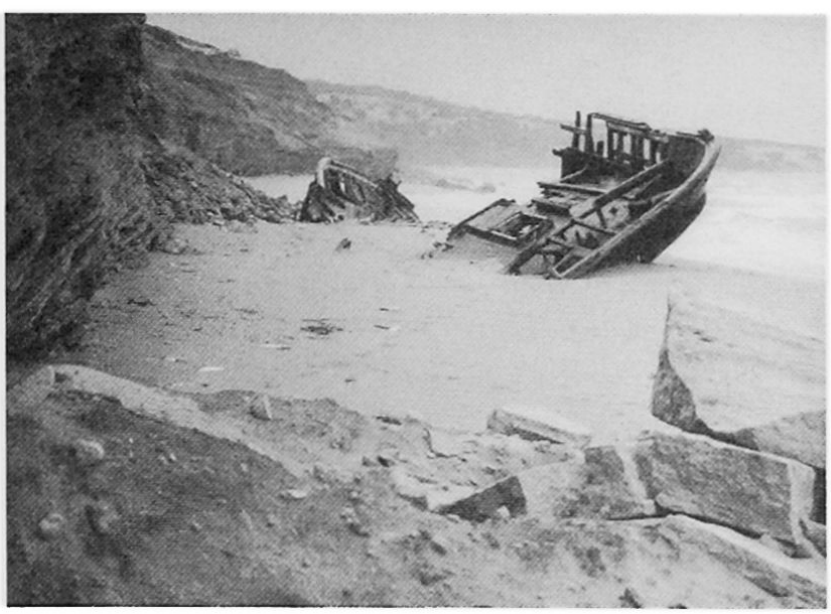

Foto 4: Kliffs westlich von Barbate.

Bei Puerto de Santa Maria $\left(6^{\circ} 25^{\prime} \mathrm{W}\right)$ mündet der im unteren Bereich bisher noch nicht überbrückbare Guadalquivir, der die Küsten über Zehner von Kilometern reichlich mit Schwemmaterial beliefert und Anlaß zur Entstehung breiter gestaffelter Dünenketten gab.

Stellenweise $a b$ der Mündung des Rio Tinto $\left(6^{\circ} 55^{\prime} \mathrm{W}\right)$ und über große Strecken ab der portugiesischen Grenze bis hinter Faro $\left(8^{\circ} \mathrm{W}\right)$ sind bei west-ost verlaufenden oder noch stärker leeseitigen Küsten
Nehrungsinseln und Strandseen entstanden, die auf reichliche Sedimentzufuhr und starke Küstenströmungen schließen lassen.

Am Cabo de São Vicente $\left(37^{\circ} \mathrm{N}, 9^{\circ} \mathrm{W}\right)$ an der Südwestspitze Portugals schließlich kommen die ersten senkrechten $60 \mathrm{~m}$ hohen Abstürze in horizontal gelagerten Kalken vor, an denen die Wellen durch keinen Strand gebremst werden und die Zerstörung durch Wellenschlag bis über $8 \mathrm{~m}$ über den Hochwasserspiegel emporreicht. Sie sind noch wesentlich stärker wellengestaltet als die Steilküsten der südlichen $\mathrm{Al}$ garve.

Bei Pedra do Cajado $\left(37^{\circ} 5^{\prime} \mathrm{N}\right)$ sind in einer Mündungsnische zwischen hohen Kliffs die ersten Strandwälle aus grobem Geröll und metergroßen Blöcken entstanden, deren Kulmination $4 \mathrm{~m}$ über dem Hochwasserniveau liegt.

Bei Carrapeteira $\left(37^{\circ} 20^{\prime} \mathrm{N}\right)$ wurden Dünenfelder im Raum einer Flußmündung aufgeweht, die den Fluß zum Umlenken zwingen (Foto 5). Die Dünen haben nicht nur die Ebene eingenommen, sondern sind auch auf die Hochplateaus über den nördlich und südlich anschließenden Kliffs verweht worden. Hier ist die erste Mündung, in die die Brandung hineinschlägt.

Für die iberischen Küsten wird zusammenfassend festgestellt, daß die atlantischen Elemente bis Gi-

Foto 5: Flußabdrängung durch Dünen bei Carrapeteira.

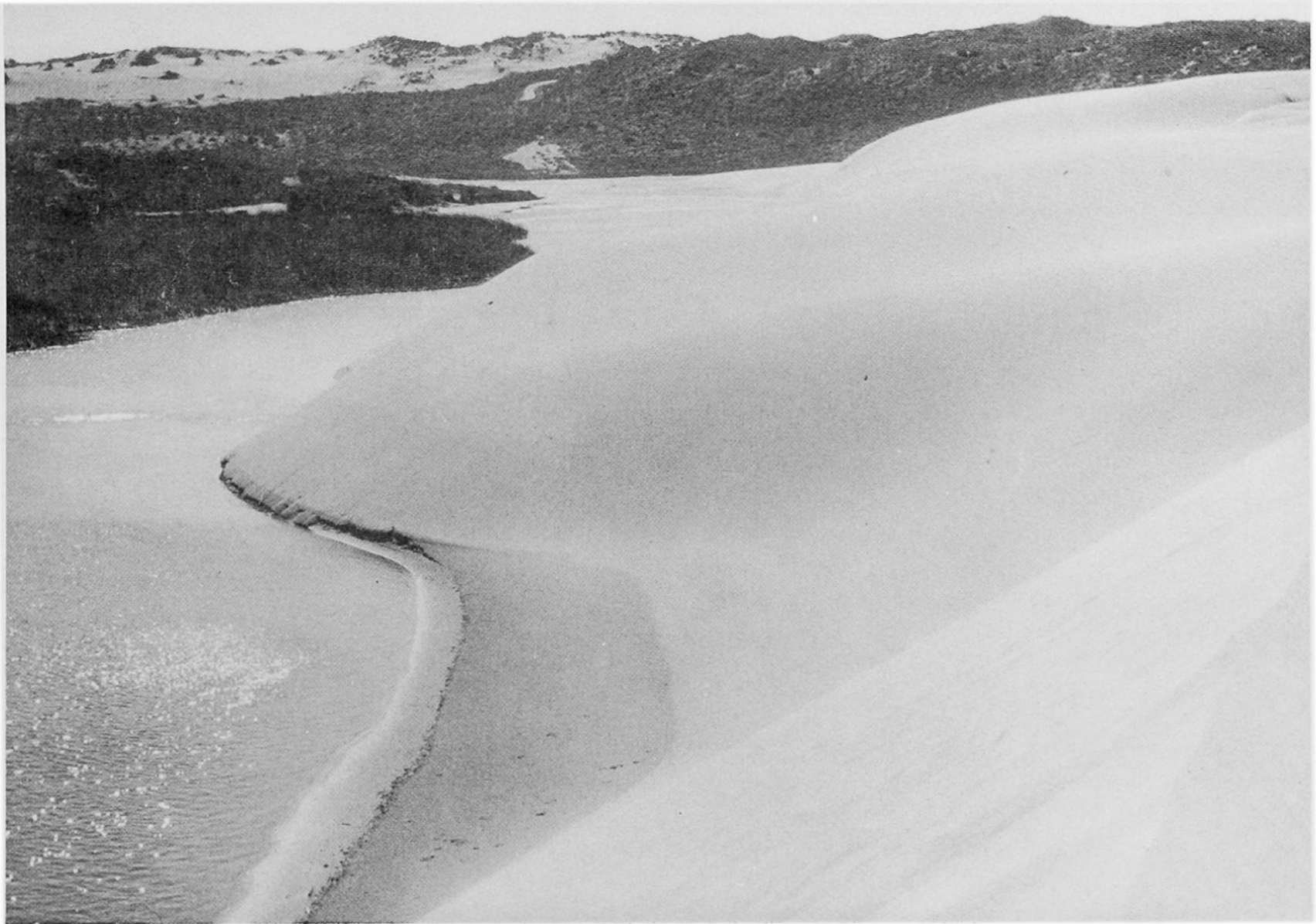


braltar kaum zu ahnen sind, bei Punta del Carnero deutlich einsetzen, ab Tarifa dominieren, vom Rio Barbate an auch die Flüsse als atlantisch anzusprechen sind und daß sich der Charakter der Küsten am Cabo de São Vicente noch einmal schlagartig verändert.

\subsection{Afrikanische Seite der Straße von Gibraltar}

Auf der afrikanischen Seite vollzieht sich der Übergang von Mittelmeer- zu Atlantikküsten in weniger Zwischenstufen.

\subsection{Südlich von Ceuta}

Außer den Hinweisen auf relativ großen Tidenhub (etwa $1 \mathrm{~m}$ ) sind die Küsten südlich von Ceuta noch ganz mittelmeerisch geprägt.

An den Steilküsten in metamorphen Gesteinen, hauptsächlich Phylliten (z. B. an der Südseite des spanischen Territoriums von Ceuta), sind zwar Kliffs entstanden, doch vollzieht sich ihre Veränderung langsam: die Pflanzendecke reicht an allen bodenbedeckten Steilhängen bis fast an die Wasserfläche hinab, die Schutthalden sind großenteils bewachsen und stabil, große Abrasionsterrassen fehlen und durch Spritzwasser hervorgerufene Lösungserscheinungen reichen nicht höher als $2 \mathrm{~m}$ über das Hochwasserniveau empor. Herabgefallene Blöcke werden nur langsam aufgearbeitet, da starker Wellenschlag selten ist und vom anfallenden Schutt werden nur die kleinen Fraktionen ins Meer oder der Küste entlang verfrachtet, denn die Küstenströmung ist schwach.

Die Steilküsten in Kalkgestein (z. B. Punta Blanca) erinnern an die schon erwähnten mittelmeerischen Kalkküsten Spaniens. Steile Hänge mit tiefen Lösungskehlen kennzeichnen sie. Schutthalden weisen an ihrem Fuß eine intensive organogene Beanspruchung auf, sind aber wenig wellengeprägt.

Die Flachküsten (südlich von Ceuta) sind durch breite flache Sandstrände charakterisiert, an denen zwar eine Küstenströmung ausgleichend wirkt, aber Brandungseinfluß kaum zu spüren ist. Strandwälle sind nur angedeutet. Die Mündungen der kleinen Bäche sind leicht nach Süden verschleppt und außer nach Regenfällen durch Strandvertriftung verschlossen. Bei stärkerer Sedimentzufuhr können instabile Deltas (Größenordnung wenige Zehner von Metern) entstehen.

\subsection{Punta Almina bis Cap Malabata}

An diesem etwa $50 \mathrm{~km}$ langen Abschnitt grenzt im Osten ein akzentuiertes quarzit- und kalkgesteinreiches, im Westen ein sanft welliges mergelig-toniges Hügelrelief an die Meerenge von Gibraltar. Die atlantischen Elemente im die Küste gestaltenden Prozeßgefüge sind weniger zahlreich und schwächer ausgeprägt als auf der gegenüberliegenden spanischen Seite, was auf die unterschiedliche Exposition der Küste zurückzuführen ist.

An den Steilabfällen der Nordseite von Ceuta sind Abrasionsplattformen häufig, erreichen aber nirgends mehr als $20 \mathrm{~m}$ Breite. Im stark geklüfteten und heterogenen Gestein wurden kleine Kanzeln und Rippen herauspräpariert. Anfallendes Feinmaterial wird nach Osten abtransportiert; verheerend wirkt sich das für die gerade östlich des Ortes am Ufer angelegte Müllkippe aus.

Weiter westlich auf marokkanischem Territorium kommen je nach Exposition zwei Steilküstentypen vor: einerseits kaum wellengeprägte Steilabstürze mit Lösungskehlen und stabil wirkenden Schutthalden, andererseits Kliffküsten mit Abrasionsflächen, die in Habitus und Dynamik denen zwischen Tarifa und Punta del Carnero entsprechen.

Zwischen Felsnasen und Hügelvorsprüngen sind in den Lockersedimenten der Buchtfüllungen mittelmeerisch anmutende flache Strände ohne starken Welleneinfluß entstanden. Die Mündungen sind kaum verschleppt; aber das dem Meer zugeführte Material wird von der Küstenströmung vollständig erfaßt und nach Osten verfrachtet.

\subsection{Cap Malabata bis Cap Spartel}

Dieser Abschnitt, der halb so lang ist wie der vorangegangene, gliedert sich in die weite Bucht von Tanger und die Steilküste westlich der Stadt bis zum Ausgang der Meerenge.

Die Bucht von Tanger bleibt im Westteil von hohem Wellengang verschont. Die Schorre besteht aus breiten Sandakkumulationen, wie sie zwischen dem Ufer und dem sanft ansteigenden Hügelland vorkommen. Ein $150 \mathrm{~cm}$ hoher Strandwall tritt im Ostteil der Bucht auf. In diesem Teil der Bucht ist auch - erstmals auf dem Wege von Osten nach Westen entlang der afrikanischen Seite der Meerenge - rasch voranschreitender Landverlust (mit nachfolgender Neuschüttung) auffallend, wie wellenzerstörte Gebäude und angegriffene neue Hotelanlagen zeigen (Foto 6). Die Mündungen der Bäche sind teilweise kanalisiert. Wo dies nicht der Fall ist, erfolgt die Verschleppung der Mündung in den feinkörnigen breiten Strandakkumulationen nach Westen auf Tanger zu, nicht nach Osten im Sinne der vorherrschenden Strömung in der Meerenge. In der Bucht ist eine rückläufige Strömung als Turbulenz entstanden, wie man sie auch in der Bahía de Algeciras erkennt.

Verglichen mit der Bucht von Algeciras ist die Bucht von Tanger stärker tide-, strömungs- und wellengeprägt. Beide aber sind weder typische Mittelmeernoch Atlantikbuchten.

Die letzten $15 \mathrm{~km}$ der Meerengenküsten sind wiederum steil. Meist bilden mächtige Blöcke und zwischengelagerte Kiesstrände das Ufer, nicht Kliffs. 


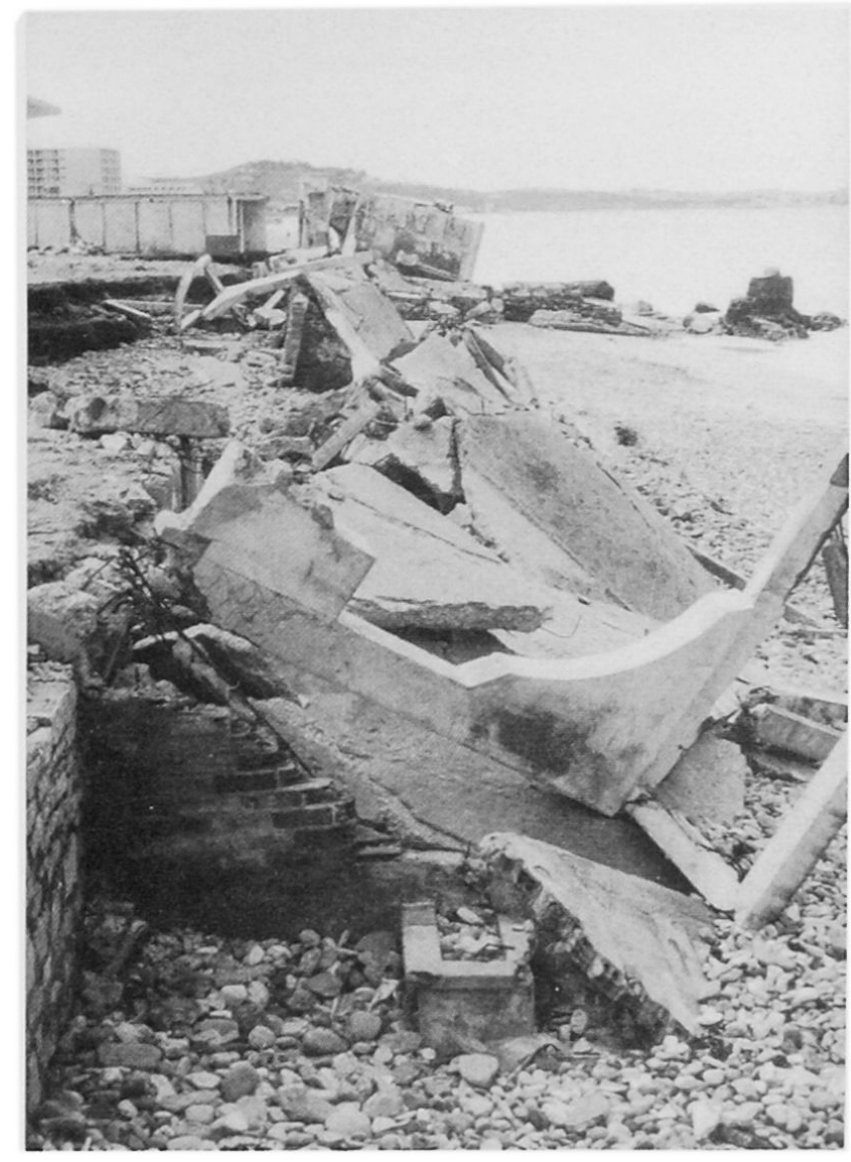

Foto 6: Zerstörte Teile neuer Hotelanlagen im Ostteil der Bucht von Tanger.

Die Macchia sitzt als dichter Filz auf allen bodenbedeckten Hangpartien und verhindert eine Zerrunsung der Hänge. Im Hangmaterial (Flysch der oberen Kreide) gehen nach längeren Feuchtigkeitsperioden im Umkreis kleiner Quellen Rutschungen und Sackungen größeren Ausmaßes nieder. Sie werden von Wellen und Küstenströmung aufgezehrt, ein aktiver Angriff erfolgt dagegen nur an einzelnen Felsnasen.

\subsection{Südlich von Cap Spartel}

Mit dem Umbiegen der Küste und der größeren Ausrichtung zur anlaufenden Brandung verändert sich die Dynamik der Steil- und eingeschalteten Flachküsten unvermittelt.

Aktive Kliffs in massigen hellen Sandsteinen (Obere Kreide), deren Maximalhöhe durch Abrasionsflächen vorgegeben sind, treten an die Stelle der großen Einzelblöcke. Rutschmassen und herabgestürzte Hangpartien werden rasch aufgezehrt und über die aufgewühlte Lockersedimentschorre nach Süden transportiert.

Wo breite Sandakkumulationen mit hohen Strandwällen den Kliff-Fuß vor dauernder Umgestaltung schützen, sind die Steilhänge mit Sandschleiern überweht und auf den Horizontalflächen sind mobile
Dünen gewachsen. Weiter gegen Süden werden flache Küsten mit stark atlantischem Gepräge (Tideeinfluß, starke Küstenströmung, hohe Brandung, heftige Deflation) gegenüber den Steilküsten vorherrschend.

Die Mündungen der Bäche gerade südlich von Cap Spartel führen nur nach Regenfällen Wasser und sind im Strandbereich nach Süden versetzt. Die weiter entfernt mündenden Flüsse (Mrarhas, Hachef, Aiacha) mit einem Gepräge, das mehr dem Rio Barbate entsprechen dürfte, wurden nicht mehr aufgesucht.

\section{Zusammenfassung}

Die südiberischen Küsten des Mittelmeeres haben ein Gepräge, das sich von dem des Atlantik stark unterscheidet. Sind auf der einen Seite nur kaum durch Tidenhub, Küstenströmung und Brandung geprägte Steilküsten mit Lösungskehlen, Schutthalden neben Flachküsten ohne starke Dynamik und episodisch bis periodisch grobe Sedimentschüttungen ins Meer führende Torrenten charakteristisch, so sind auf der anderen Seite stark von der Meeresseite her gestaltete Küsten kennzeichnend, die in den steilen Abschnitten viele aktive Kliffreihen, in den flachen breite dynamische Sandstrände mit Dünen zeigen und wo durch kontinuierlich mit Wasser gefüllte Flußbetten große Mengen feinkörnigen Materials dem Meer zugeführt werden.

Der Übergang zwischen Mittelmeer und Atlantik vollzieht sich im Grenzsaum der Meerenge von Gibraltar. Bei einem Gang von Osten nach Westen auf der europäischen und afrikanischen Seite werden die einzelnen Stufen der Veränderungen auf die Küstenformen und die rezente Morphodynamik aufgezeigt.

Êinerseits ist die vorliegende Arbeit regional ausgerichtet. Andererseits will sie einen Beitrag zu einem in der Morphologie wenig beachteten Problemkreis liefern, nämlich zur Frage, wie ein Grenzsaum zwischen zwei geomorphologisch gänzlich unterschiedlichen Bereichen in seiner Differenzierung erkannt, erklärt und vereinfachend dargestellt werden kann.

\section{Literaturverzeichnis (ohne Karten)}

BUEN, R. de (1932): Contribución al estudio del relieve del Estrecho de Gibraltar. Campañas del Xauen en 1932. In: Notos y Resúmens Inst. espan. oceanogr., ser. II/66, 1-15.

Deutsches Hydrographisches Institut (1959): Mittelmeer-Handbuch, 1. Teil, Nr. 2027, Hamburg.

GIERMANN, G. (1962): Meeresterrassen am Nordufer der Straße von Gibraltar. - In: Ber. Naturf. Ges. Freiburg i. Br., 52, 111-118. 
HERNANDEZ-PACHECO, F. (1961): Rasgos geográficos y geológicos del Estrecho de Gibraltar y de las comarcas que lo limitan. - In: Re. R Acad. Cienc., 55, 105-139.

HOUSTON, J.M. (1964): The western Mediterranean world. An introduction to its regional landscapes. London.

JESSEN, O. (1927): Die Straße von Gibraltar. - Berlin. LAUTENSACH, H. (1928): Morphologische Skizze der Küsten Portugals. Ein landeskundlicher Ausschnitt. - In: Sonderbd. Z. Ges. Erdkunde Berlin, Hundertjahrfeier, 296-340.

LAUTENSACH, H. (1955): Der Rhythmus der Jahreszeiten auf der Iberischen Halbinsel. - In: Geogr. Rundschau 7, 1-12.

\section{Literaturbesprechung}

MONHEIM Rolf: Fussgängerbereiche und Fussgängerverkehr in Stadtzentren in der Bundesrepublik Deutschland. Bonner Geographische Abhandlungen Heft 64. 290 S., 56 Abb., 46 Tab. Geogr. Institut der Universität Bonn, 1980. DM 36.---

Schon durch eine ganze Reihe früherer Veröffentlichungen ist MONHEIM als ein hervorragender Kenner der Entwicklung und Problematik von Fussgängerbereichen in deutschen Innenstädten bekannt. Die hier zu besprechende Druckfassung seiner im Original noch wesentlich umfangreicheren Habilitationsschrift hat das erklärte Ziel, Fussgängerbereiche unter Beschränkung auf die "normativ-konzeptionellen Gesichtspunkte der Innenstadtentwicklung" (S.5) darzustellen, wobei das Schwergewicht in der Untersuchung des Fussgängerverkehrs selbst liegt, der als "Spiegel der Funktionsweise von Fussgängerbereichen" (S.5) aufgefasst wird.

Die Arbeit versteht sich als ein Beitrag zur Angewandten Geographie, dessen Grundorientierung vom Verfasser selbst (im Gegensatz zu einigen pessimistisch-kulturkritischen Abhandlungen anderer Autoren) eher als "optimistisch-reformerisch bemüht" charakterisiert wird. Bei der insgesamt positiven Einstellung gegenüber den durch die Errichtung von Fussgängerbereichen ausgelösten Prozessen geht es MONHEIM darum, solche Entwicklungen bewusst zu machen, die auf einen "menschengerechten Lebensraum" hinzielen, sowie Fehleinschätzungen offenzulegen, die diese Entwicklung bis heute bei der Innenstadtplanung behindern (S. 18). Die Grundlagen der Arbeit bilden einerseits unfangreiche Erhebungen des Passantenverkehrs (Passantenzählungen und -befragungen) in verschiedenen Städten, die ergänzt werden durch Expertenbefragungen sowie eine Verwaltungsumfrage bei allen Städten und Gemeinden der Bundesrepublik, die zur Zeit der Erhebungen über eigene Fussgängerbereiche verfügten; sehr intensiv in die Untersuchung mit einbezogen wer-
LAUTENSACH, H. (1964): Iberische Halbinsel. - München.

Naval weather service detachment (1975): A climatic résumé of the Mediterranean Sea. - Acheville NC.

PARDÉ, M. (1964): Les régimes fluviaux de péninsule iberique. - In: Rev. Geogr., 39, 129-182. Lyon.

SAENZ GARCIA, C. (1959): Peculiaridades morfológicas des los ríos españoles. - In: Bol. Real. Soc. Geogr., 95, 223-238. Madrid.

TERÁN, M. de (ed.) (1952): Geografía de España y Portugal. - Bd. 1 und 2. Barcelona.

den darüber hinaus die Ergebnisse einer Fülle weiterer empirischer Forschungen, die grösstenteils erst im Verlauf der Arbeit, einige sogar erst nach Abschluss des Habilitationsverfahrens (1976) erschienen sind.

Das erste Hauptkapitel befasst sich mit der generellen Entwicklung deutscher Innenstädte und deren Bedeutung verschiedener Fussgängerbereichs. konzeptionen im Rahmen dieser Entwicklung. Die weiteren Kapitel sind quantitativen Aspekten von Passantenstruktur und -verhalten in Fussgängerbereichen gewidmet (Entwicklung der Passantenzahlen, Tätigkeiten der Besucher, Verkehrsmittelwahl beim Weg zur Innenstadt sowie der Analyse der Wege beim Innenstadtbesuch selbst). Das Hauptgewicht liegt hierbei in der systematisierenden und typisierenden - für eine Uebersichtsarbeit oft ungewöhnlich detaillierten Deskription eigener oder von anderer Seite erhobener Daten. Dabei bietet die Arbeit einen sehr guten Ueberblick über die bisher für Untersuchungen des Passantenverkehrs in Fussgängerbereichen entwickelten Methoden; deren Beurteilung im Hinblick auf Zuverlässigkeit und Gültigkeit hätte freilich manchmal kritischer ausfallen können. Die Verwendung derart unterschiedlich entstandener Daten zum Zwecke des zeitlichen oder räumlichen Vergleichs erfordert daher einen gewissen Mut, der sich allerdings bei der "explorativ-hypothensbildenden Zielsetzung" der Studie (S.19) durchaus rechtfertigen lässt. Auch wenn einige Skepsis bezüglich mancher quantitativer Ergebnisse der Arbeit bliebe, so sollte dies keinesfalls den Blick darauf verstellen, dass diese Untersuchung sehr viele interessante Denkanstösse für die Praxis enthält, die schliesslich in ein Plädoyer für möglichst umfassende und komplexe Fussgängerbereichsplanungen münden.

GH $3 / 81$ Walter Kuhn, München 